\title{
Programa Interunidades de \\ Doutoramento em Enfermagem: \\ 21 anos construindo ciência
}

DOCTORING INTERUNITS IN NURSING PROGRAM: 21 YEARS BUILDING SCIENCE

PROGRAMA INTERUNIDADES DE POSTGRADO EN ENFERMERÍA:

21 AÑOS CONSTRUYENDO LA CIENCIA

\author{
Isília Aparecida Silva ${ }^{1}$, Maria das Graças Carvalho Ferriani ${ }^{2}$, Emília Campos de Carvalho $^{3}$
}

\begin{abstract}
RESUMO
O Programa Interunidades de Doutoramento em Enfermagem foi o primeiro a ser criado na América Latina e titulou 290 enfermeiros, até o primeiro semestre de 2004. Este estudo apresenta algumas características das teses defendidas no programa, focalizando as áreas temáticas e métodos de pesquisa utilizados. Foram analisados os resumos de 287 teses, defendidas até o primeiro semestre de 2004. As áreas mais investigadas, até o momento foram: Saúde da Criança, da Mulher, do Adulto/ Idoso e Educação. O método de pesquisa quantitativo predominou até final da década de 1980 , havendo uma intensa produção de pesquisas no método qualitativo a partir desse período. Ainda na primeira década do programa, os temas passaram a versar sobre os aspectos que relacionam a prática da enfermagem aos contextos políticos e sociais, convergindo para o atual eixo paradigmático do programa: "Bases teóricas, filosóficas, históricas do saber e da prática de enfermagem”.
\end{abstract}

\section{DESCRITORES}

Educação de pós-graduação em enfermagem.

Pesquisa em enfermagem.

Pesquisa em avaliação de

enfermagem

\begin{abstract}
The Doctoring Interunits in Nursing Program was the first created in Latin America and has titled 290 nurses until the second semester of 2004. This study presents some thesis characteristics upheld in the program, focusing the thematic areas and research methods used. The summaries of 287 theses supported until the first semester of 2004, have been analyzed. The most investigated fields up to now were: Children, Woman, Adult/ Elderly Health and Education. The quantitative research method predominated until the end of the 80 's, since then an intense production of qualitative method researches started to occur. Still in the first decade of the program, the themes started to talk about the aspects that connect the practice of nursing to the political and social contexts, converging to the current program's paradigmatic axis: "Theoretical, philosophical, historical bases of knowledge and of the nursing practice.”
\end{abstract}

\section{KEY WORDS}

Education, nursing, graduate. Nursing research.

Nursing evaluation research

\section{RESUMEN}

El Programa Interunidades de Doctorado en Enfermería fue el primero en ser creado en América Latina habiendo graduado a 290 enfermeros, hasta el primer semestre del 2004. En este estudio se presenta algunas características de las tesis sustentadas en el programa, enfocando las áreas temáticas y métodos de investigación utilizados. Fueron analizados los resúmenes de 287 tesis, sustentadas hasta el primer semestre del 2004. Las áreas más investigadas, hasta el momento fueron: Salud del Niño, de la Mujer, del Adulto/Anciano y Educación. El método de investigación cuantitativa predominó hasta fines de la década de 1980, habiendo una intensa producción de investigaciones en el método cualitativo a partir de ese período. Aún en la primera década del programa, los temas pasaron a versar sobre aspectos que relacionan la práctica de la enfermería a los contextos políticos y sociales, convergiendo hacia el actual eje paradigmático del programa: “Bases teóricas, filosóficas, históricas del saber y de la práctica de enfermería”.

\section{DESCRIPTORES}

Educación de postgrado en enfermería.

Investigación en Enfermería. Investigación en evaluación de enfermería.
1 Professora Titular do Departamento Enfermagem Materno-Infantil e Psiquiátrica da Escola de Enfermagem da Universidade de São Paulo(EEUSP). isasilva@usp.br

2 Professora Titular do Departamento Enfermagem Materno-Infantil e Saúde Pública da Escola de Enfermagem de Ribeirão Preto da USP (EERP-USP)

3 Professora Titular do Departamento Enfermagem Geral e Especializada da EERP-USP. 
Isília Aparecida Silva

Maria G. C. Ferriani

Emília C. de Carvalho

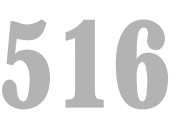

Rev Esc Enferm USP 2005; 39(Esp.):515-21.

\section{INTRODUÇÃO}

Na era da globalização, um dos temas polêmicos, principalmente no meio acadêmico, diz respeito à avaliação da produção científica. E, particularmente nos interessa a produção científica na área da saúde.

As vertentes de pensamento crítico sobre a questão, apontam, cada vez mais, para a necessidade de uma avaliação qualitativa da produção, em que pese sua contribuição em um plano geral para a humanidade, ou na contribuição para as transformações de condições de vida e de saúde da população, de interesse local ou regional.

No entanto, ainda parece distante o tempo em que os indicadores bibliométricos e fatores de impacto de publicação, adotados amplamente no meio científico para metrificar e qualificar a produção cederão espaço ou possam conviver sinergicamente com outros indicadores qualitativos, para evidenciar a qualidade e natureza do conhecimento produzido e publicado pelos pesquisadores.

A maior dificuldade talvez seja a de avaliar os trabalhos científicos, não apenas no seu aspecto de desenvolvimento teórico-metodológico e de resultados obtidos, mas do seu impacto na geração de ciência e de tecnologia, capaz de gerar novos produtos e assegurar a transferência de seus resultados, de modo efetivo e produtivo para a sociedade.

Nesse contexto de avanços, desafios e enfrentamento dos processos de avaliação da produção é que tem se inserido o Programa Interunidades de Doutoramento em Enfermagem, criado e mantido pela Escola de Enfermagem da USP e Escola de Enfermagem de Ribeirão Preto da USP, tendo sido o primeiro programa de pós-graduação, nível doutorado na América Latina, e responsável pela titulação de 290 doutores, até julho de 2004.

Em que pese os paradigmas atuais e suas tendências em avaliação da produção científica é indiscutível a contribuição de um programa desse porte para a construção do conhecimento da área da Enfermagem, tanto do ponto de vista da produção em si, representada pelas teses defendidas como trabalho de conclusão de curso, quanto da formação de recurso humano multiplicador do conhecimento e qualificação de profissionais para o desenvolvimento da atividade de pesquisa.

A enfermagem, assim como toda área do conhecimento, tem buscado na pesquisa, os instrumentos para assegurar seu campo de atuação e configurar um saber próprio, sendo a atividade de investi- gação científica fortalecida e melhor instrumentalizada com as grandes descobertas tecnológicas que culminaram, nas últimas décadas do século XX, com o desenvolvimento de recursos eletroeletrônicos que propiciaram avanços antes não imagináveis para a ciência.

A apropriação da atividade de pesquisa como ação da enfermagem é relativamente recente, sendo que os registros históricos descrevem o "Censo de 1950" como o primeiro trabalho científico realizado por enfermeiras ${ }^{(1)}$.

A pesquisa em enfermagem, na década de 1950 teve seu início fortemente marcado pela influência da Associação Brasileira de Enfermagem, ABEn, e de Escolas de Enfermagem, cujas temas versaram sobre o ensino e a profissão. Mas, foi a partir da década de sessenta, com a criação dos cursos de Graduação em Enfermagem no nível superior, que se percebe incremento da produção científica de enfermeiras, a primeira tese defendida por enfermeira, apresentada para concurso de Professor Catedrático, na Universidade de São Paulo,USP, na década de $1960^{(2)}$. Também, os temas de eventos científicos da área, abordaram com freqüência a relação da pesquisa e enfermagem, a exemplo do XVI Congresso Brasileiro de Enfermagem, sediado em Salvador, em 1964 ${ }^{(3)}$.

No primeiro lustro da década de 1970, com a criação dos cursos de mestrado, na Escola Anna Nery, da Universidade Federal do Rio de Janeiro e Escola de Enfermagem da USP, a atividade de pesquisa toma o impulso para a construção do saber próprio da Enfermagem ${ }^{(4)}$, seguidos, como já referido, pela criação do Programa Interunidades. Esses programas podem ser considerados bases iniciais para a consolidação do papel da pós-graduação no desenvolvimento da pesquisa e na formação de pesquisadores da área no Brasil, e que nos leva a afirmar que temos, no conjunto dos 24 Programas de Pós-Graduação em Enfermagem ${ }^{(4)}$,hoje, um núcleo forte de construção do conhecimento em enfermagem.

É nesse contexto, que um olhar, mesmo que panorâmico, sobre as pesquisas que constituíram as teses de alunos de doutoramento, do Programa Interunidades, propicia uma reflexão sobre a contribuição desses estudos para o saber da enfermagem. Dessa forma, este estudo tem por objetivo descrever algumas características dessa produção, focalizando as áreas temáticas mais freqüentemente pesquisadas ao longo dos 22 anos de existência do programa e as abordagens metodológicas utilizadas nesses estudos. 


\section{DESENHO DO ESTUDO}

Trata-se de um estudo descritivo de natureza quantitativa, cujos dados foram coletados a partir de resumos das teses defendidas pelos alunos do Inter, como costumamos nos referir ao Programa Interunidades de Doutoramento em Enfermagem, de 1983, data da primeira tese defendida até o final do primeiro semestre de $2004^{(5)}$.

Os resumos foram xerocopiados das teses e os dados, levantados a partir da leitura dos mesmos, foram registrados em um instrumento criado para essa finalidade onde registrou-se o ano de defesa da tese, o nome do autor, objeto de estudo, área temática em que o estudo se insere e referencial teórico e metodológico utilizado para a realização da pesquisa. As teses foram identificadas a partir de uma listagem obtida do sistema Fênix ${ }^{(5)}$ da Universidade de São Paulo e localizadas na Biblioteca da Escola de Enfermagem da USP, sede do acervo de teses do Inter.

Os objetos de estudo foram agrupados por áreas temáticas elaboradas para essa finalidade, considerando a concepção tradicional de áreas de conhecimento na enfermagem, cuja denominação tem sido comumente encontrada em disciplinas e áreas de concentração dos programas de pós-graduação em enfermagem, no último triênio ${ }^{(4)}$ : Saúde da Criança e do adolescente, Saúde da Mulher, Saúde Coletiva, Saúde Mental e Psiquiátrica, Educação, Gerenciamento, Saúde do Idoso, Saúde do Trabalhador, Informação e comunicação e outros. Os estudos foram agrupados segundo essas áreas e aquelas cuja freqüência não ultrapassou $2 \%$ do total das teses avaliadas, ou cujo objeto de pesquisa versava sobre tema geral para a enfermagem, sem ater-se a uma área específica, foram agrupados na categoria outros.

\section{APRESENTAÇÃOE DISCUSSÃO DOS RESULTADOS}

A primeira tese defendida por aluno do Programa Interunidades, foi em 1983, intitulada "Flora microbiana da boca e chupeta de criança hospitalizada com desidratação”, cuja autora foi a Professora Maria Helena Marques, então docente da Escola de Enfermagem da USP. Desde essa tese, até o final do primeiro semestre de 2004, soma-se o total de 290 defesas realizadas no Inter.

Dentre as 290 teses, não foi possível localizar três delas, cujos exemplares, embora tenham sido cadastrados na biblioteca, não foram localizados até o momento da conclusão do estudo, totalizando assim, 287 resumos de teses analisados.

\section{AS ÁREASTEMÁTICASESTUDASNO INTER}

Tomamos como base para a análise das teses, em uma primeira instância, as áreas temáticas focalizadas pelos objetos de estudo da pesquisa, tal qual ele se apresentava, ou como mais se aproximava, a partir de nossa interpretação, uma vez que muitos resumos não explicitavam o objeto ou mesmo a justificativa da investigação. Essas áreas constituíramse as categorias para análise desse trabalho.

Assim, as 287 teses foram distribuídas em dez áreas temáticas: Saúde da Criança e do adolescente, Saúde da Mulher, Saúde do Adulto / Idoso, Saúde Coletiva, Saúde Mental, Gerenciamento, Educação, Informação e Comunicação, Saúde do Trabalhador e Outros. Os dados foram analisados ano a ano, e em alguns aspectos, foram agrupados em décadas para melhor explicitação gráfica para apresentação dos resultados.

Pelos dados apresentados na Tabela 1 , é possível observar que as áreas que tiveram o maior número de teses vinculadas ao longo dos 22 anos do Programa, foram Saúde da Mulher, 46 (16\%), Saúde do Adulto/Idoso, 46 (15,7\%), Educação 42 (14,7\%), Gerenciamento 38 ( 13,25\%), Saúde da Criança e do Adolescente 32 (11,14\%), Saúde Coletiva 24 (8,4\% ), Saúde Mental 23 ( 8\%), Informação e Comunicação 10 ( 3,48 \%), Saúde do Trabalhador 8 (2,78\%) e Outros com $19(9,4 \%)$.

A Tabela 1 permite observar a evolução do desenvolvimento das pesquisas segundo a área temática, no qual está demonstrado que as áreas de Saúde da Mulher, Saúde da Criança, Gerenciamento, Saúde do Adulto/Idoso e Educação, embora tenham uma freqüência relativa variada entre si, mantiveram uma regularidade de desenvolvimento de estudos, tendo pelo menos uma pesquisa a cada três anos, o que significa que em todo o percurso do programa, sempre teve algum orientador/aluno vinculados ao tema, se considerarmos o tempo de titulação, que até meados do final da segunda década do programa, era em média de 50 meses.

Considerando que a primeira etapa do programa, aqui demarcada na década de 1980 - 1989, foram defendidas um total de 37 (100\%) teses, vamos perceber que a área da Saúde do Adulto / Idoso com 7 $(18,9 \%)$ teses, Gerenciamento 7 (18,9\%) e Educação $5(13,5 \%)$ foram as mais estudas nesse período, seguidas por Saúde da Criança com 4 (10,8\%) das teses e Saúde da Mulher 3 (8,1\%). A segunda década, aqui considerada, a freqüência apresentada por essas áreas, com base no total de 169 (100\%) teses para o período de 1990-1999, evidencia que a área da Saúde da Criança com 19 (11,24\%) teses, mante-
Programa Interunidades de Doutoramento em Enfermagem: 21 anos construindo ciência 
Isília Aparecida Silva Maria G. C. Ferriani Emília C. de Carvalho ve um percentual próximo da primeira década, mesmo apresentando um aumento de teses com essa temática. Enquanto as áreas de Saúde da Mulher 24 (14,2\%), Saúde Coletiva 13 (7,7\%) e Saúde Mental e Psiquiátrica 15 (8,87\%), tiveram um grande impulso com aumento expressivo de teses para essa década, o mesmo não podendo ser afirmado para a área de Saúde do Adulto / Idoso 23 (13,6\%) e a área Geren- ciamento, $23(13,6 \% \%)$ e que tiveram um decréscimo percentual, relativo ao total de teses para a década. Chama a atenção que para esse período, a área Educação 26 (15,38 \% ), também teve um aumento significativo, podendo ser associada aos movimentos de reformulação de currículos de graduação que ocorreram no final da década de 80 e princípios de década de 90.

Tabela 1. Freqüência das teses segundo as áreas temáticas e década. Programa Interunidades de Doutoramento em Enfermagem, EEUSP/EERPUSP, 2004 - São Paulo, 2004.

\begin{tabular}{lcccc}
\hline Áreas & $\mathbf{1 9 8 3 - 1 9 8 9}$ & $\mathbf{1 9 9 0 - 1 9 9 9}$ & $\mathbf{2 0 0 0 - 2 0 0 4}$ & Total \\
\hline Saúde da Criança & $4(10,8 \%)$ & $19(11,24 \%)$ & $9(11,1 \%)$ & $32(11,14 \%)$ \\
Saúde da Mulher & $3(8,1 \%)$ & $24(14,2 \%)$ & $19(23,4 \%)$ & $46(15,7 \%)$ \\
Saúde do Adulto-Idoso & $7(18,9 \%)$ & $23(13,6 \%)$ & $16(19,7 \%)$ & $46(15,7 \%)$ \\
Saúde Coletiva & $2(5,4 \%)$ & $13(7,7 \%)$ & $9(11,1 \%)$ & $24(14,5 \%)$ \\
Saúde Mental e Psiquiátrica & $2(5,4 \%)$ & $15(8,87 \%)$ & $6(7,4 \%)$ & $23(8,01 \%)$ \\
Gerenciamento & $7(18,9 \%)$ & $23(13,6 \%)$ & $7(8,6 \%)$ & $37(12,89 \%)$ \\
Educação & $5(13,5 \%)$ & $26(15,3 \%)$ & $11(13,5 \%)$ & $42(14,63 \%)$ \\
Informação e Comunicação & $3(8,1 \%)$ & $6(3,55 \%)$ & $1(1,23 \%)$ & $10(3,48 \%)$ \\
Saúde do Trabalhador & $2(5,4 \%)$ & $5(2,95 \%)$ & $1(1,23 \%)$ & $8(2,78 \%)$ \\
Outros & $2(5,4 \%)$ & $15(8,87 \%)$ & $2(2,4 \%)$ & $19(6,62 \%)$ \\
\hline Total & $37(\mathbf{1 0 0 \% )}$ & $\mathbf{1 6 9 ( 1 0 0 \% )}$ & $\mathbf{8 1 ( 1 0 0 \% )}$ & $\mathbf{2 8 7 ( 1 0 0 \% )}$ \\
\hline
\end{tabular}

Ao visualizarmos a terceira década, de 2000-2004, do total de 81 (100\%) teses, as áreas com maior freqüência de teses para esta década foram Saúde da Mulher 19 (23,4\%) e Saúde do Adulto / Idoso 16 (19,75\%) e Educação 11 (13,5\%), sendo essas, também, as que apresentaram o maior número de investigações no período total, 46 (15,7\%) , 46 (15,7\%) ) e 42 (14,5\%), respectivamente.

Embora o quantitativo das teses, distribuídas ao longo dos 22 anos do Programa, possa demonstrar que as áreas tiveram incremento importante no período de 1990-1999, a freqüência relativa de algumas áreas se mantiveram praticamente constantes. A única que apresentou crescimento contínuo foi a área da Saúde da Mulher e Saúde Coletiva.

A área de Saúde Coletiva, só mantém uma regularidade de produção de teses a partir de 1992, quando a partir daí, também demonstra um incremento significativo, passando de duas teses defendidas (5,4\% ) na década de 80 , para $13(7,7 \%$ ) e nove $(11,1 \%$ ) na década de 1990 e primeira metade da década de 2000, respectivamente.

A área de Saúde Mental e Psiquiátrica também apresentou um número menor de teses na primeira década do Programa, passando a um incremento significativo para a segunda, voltando a declinar posteriormente nos anos 2000-2004.

A freqüência dos objetos de estudo, relacionado às áreas temáticas, parece estar associada ao foco de interesse e atuação do orientador. Em tempos iniciais do programa, ainda na década de 1980, em sua maioria, a escolha do orientador não se pautava na temática que esse desenvolvia como eixo principal de sua produção. Frente a necessidade de absorver a demanda que se apresentava, o tema a ser desenvolvido pelo aluno não era critério de vinculação ao orientador, desde que a metodologia fosse de seu domínio, principalmente, considerando o ecletismo do corpo docente e a diversidade de sua origem, pois grande parte deles pertenciam a outras unidades da USP não sendo enfermeiros ou docentes de uma das Escolas de enfermagem sedes do programa.

A partir do final dos anos 90, frente às exigências estabelecidas pelo novo paradigma que passou a sustentar o sistema de avaliação dos Programas de Pós-graduação, pela CAPES ${ }^{(6)}$, Coordenadoria de Aperfeiçoamento do Ensino Superior, passou-se a exigir uma organização mais explicita dos projetos e linhas de pesquisa, o que se fez sentir também, na necessidade de vinculação dos projetos de teses aos projetos dos docentes, implicando em um alinhamento da produção docente e discente, impingindo o seguimento de caminhos temáticos pelo candidato ao Programa, trilhado pelo possível orientador. Assim, o aumento ou diminuição da freqüência das teses segundo a temática, não significa, necessariamente menor interesse ou perda do grau de prioridade, destas. Inferimos que a partir dos anos 90, há um aumento
Rev Esc Enferm USP 2005; 39(Esp.):515-21. 
de orientadores no programa que pertenciam ao quadro docente das duas unidades egressos do Inter, que passam a trabalhar questões voltadas para suas áreas de especialização, induzindo objetos de pesquisa dentro de temáticas de interesse de suas áreas de atuação. Podendo assim, esperar-se um movimento de fluxo variável entre as temáticas, e mesmo a inserção de uma nova área, que se consolida ao longo de um período, como é o caso de Saúde do Trabalhador, que mantém uma freqüência relativa de teses, provavelmente dependente de maior número de orientadores de desenvolvam a temática. Essa área no programa Interunidades emerge em 1987. De acordo com Oliveira \& Lisboa ${ }^{(7)}$, a temática saúde do trabalhador, como campo de conhecimento no Brasil, inicia com estudos a partir da década de 80, em compasso com tendências no mundo ocidental e a transição democrática em nosso país.

Houve também, o surgimento de temas, cujo significado e tendência dentro do setor saúde, poderiam ser considerados como outras áreas temáticas, mas que, para esse estudo, a fim de facilitar a demonstração da concentração e dispersão temática, foram incorporadas nas categorias elegidas para esta apresentação. Trata-se de temas como a Saúde do Idoso, que incluímos na área Saúde do Adulto, e que desde a primeira tese em 1990, apresentou com relativa freqüência, pelo menos uma tese/ano até 2001. Outro tema, Saúde da Família, traz uma inovação para a área da Saúde da Criança, atentando para a vivência de pais e familiares frente a fase e condições do processo de saúde e de doença da criança, ampliando o foco de atenção dos profissionais dentro dessa área.
No final da década de 1980, é possível observar que muitos dos temas de investigação em enfermagem, têm seu nível de prioridade mantidos ao longo do tempo, ocorrendo apenas a transformação das abordagens e perspectivas com que são tratados. A autora é de acordo de que essa tendência traz a possibilidade de dar respostas mais completas para dado fenômeno frente aos avanços científicos e sociais que naturalmente devem ocorrem ${ }^{(8)}$.

É possível, assim, que o panorama de algumas das áreas temáticas, que se apresentam em declínio quantitativo de teses a partir de 2000, para o Inter, esteja relacionado ao desligamento de parte de docentes de determinados domínios temáticos.

Quanto ao método de pesquisa adotado nas teses do Inter, observa-se pelos resultados expostos na Tabela 2 que o método positivista predominou até final da primeira década do Programa, sendo o ano de 1989, quando se dá o ponto de inflexão, e o método qualitativo passa a ser regular e continuamente, mais utilizado nas pesquisas, embora em 1985, 4 (60\%) das teses, daquele ano tenham sido realizadas com alguma abordagem de pesquisa qualitativa. Do total de 287 (100\%) teses, 194 $(67,6 \%)$ utilizaram alguma abordagem do método qualitativo de pesquisa e 84 (29, 26\%) em método quantitativo. Do total de teses, 11 (3,9\%) não foram classificadas, quanto ao quesito método, referencial teórico metodológico e estratégia de análise de pesquisa, por falta de clareza da redação do resumo, que impossibilitou a compreensão destes aspectos.

Tabela 2 - Freqüência das teses segundo os métodos de pesquisa e o ano. Programa Interunidades de Doutoramento em Enfermagem, EEUSP/EERPUSP, 2004 - São Paulo, 2004.

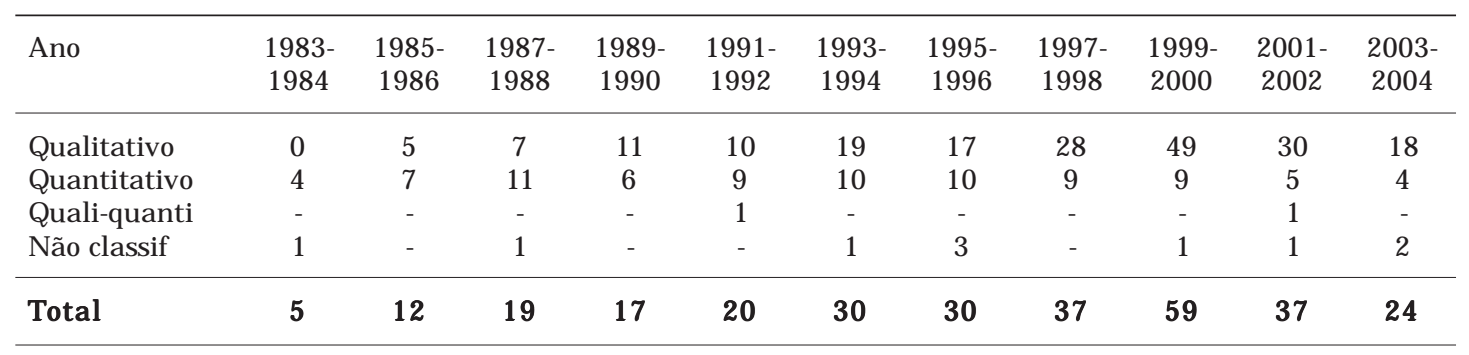

Embora desde 1985, reconheça-se a presença do método qualitativo nas pesquisas do Inter, inferimos que o I Encontro Interamericano de Pesquisa Qualitativa em Enfermagem, promovido por docentes da Escola de Enfermagem da USP e do Departamento de Enfermagem da Universidade Federal de Santa Catarina, influenciou significativamente os enfermeiros na definição e percepção de objetos de estudo, apontando para novos caminhos metodo- lógicos e novas possibilidades de dar à antigas e novas indagações. Observa-se nos anos seguintes, que a diferença entre as duas vertentes metodológicas passa a ser cada vez maior, com predomínio do método qualitativo a partir da década de 90.

Embora seja possível considerar que um evento científico possa constituir em elemento de motivação para adoção do método qualitativo para as pesquisas em enfermagem, somos acordes com demais
Programa Interunidades de Doutoramento em Enfermagem: 21 anos construindo ciência 
Isília Aparecida Silva Maria G. C. Ferriani Emília C. de Carvalho

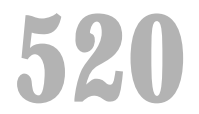

Rev Esc Enferm USP 2005; 39(Esp.):515-21. pesquisadores na área, que a utilização dos referenciais teóricos das ciências humanas, contrapondo-se aos fundamentos metodológicos das ciências naturais, que predominou por longo período, foi resultado da percepção dos pesquisadores, que o método positivista não possibilita a investigação de determinados objetos que exigem uma compreensão contextual e social. Assim, segundo Sauer ${ }^{(9)}$ as abordagens metodológicas das ciências humanas possibilitam a compreensão da experiência humana que passa a ser objeto de atenção da enfermagem, de forma mais integral desde a década de 1980, intensificando na década seguinte. Esse fenômeno se reflete também nas teses defendidas no Inter, que desde sua primeira década, demonstra a tendência de confluir sua produção para uma vertente de estudos teóricos, filosóficos e históricos da prática da enfermagem.

Ao estudar as teses do defendidas no Inter, de 1983-1990, é possível verificar que “todas explicitam um marco teórico que, para fins de estudo foi classificado segundo a diversidade de sua origem” e no conjunto das teses, a utilização dos referenciais teóricos demonstrava o "predomínio das Ciências Humanas e Sociais sobre as demais, como fonte do referencial utilizado” pelos pesquisadores ${ }^{(10)}$.

O método fenomenológico, tem sido um dos mais aplicados em teses do Programa, seguidos de teorias como Interacionismo Simbólico, Representação Social, Materialismo Histórico Dialético. Ainda, o método etnográfico e teorias de enfermagem de Orem, Wanda Horta e King. Nos estudos sobre educação, Paulo Freire aparece como importante referencial teórico. Alguns estudos recentes, na década de 2000, utilizam constructos teóricos de autoria de orientadores do programa.

Também, a análise dos resumos, demonstra que a produção do Inter foi configurada, na primeira década, a partir de objetos de estudo que ainda se atinham aos aspectos tecnológicos e funcionais da assistência, detendo-se também, nos estudos sobre atuação e definição do papel profissional, em todas as áreas temáticas, porém mais freqüente na área de gerenciamento. Da mesma forma, todas as áreas aqui consideradas com o maior número de teses vinculadas, se ocuparam em investigar sobre os determinantes do processo saúde-doença em suas especialidades, em princípio com um cunho exploratório e positivista. Porém, os objetos passam a ser definidos em uma perspectiva de compreensão da experiência humana, voltados para o desenvolvimento epistemológico do saber em enfermagem de maneira a aprofundar os aspectos que relacionam a prática da enfermagem aos contextos políticos e sociais. Essa tendência se anuncia em teses no final da década de 80 e se fortalece a partir do primeiro terço da década de 90 , com estudos que não só trazem a compreensão dos fenômenos tratados pela enfermagem, em uma perspectiva compreensiva, interpretativa, analítica e crítica, mas tem como resultados, constructos teóricos que colaboram na construção do conhecimento e explicação do comportamento social.

Se considerarmos a atual configuração do Programa Interunidades, cuja nucleação central é “Bases teóricas, filosóficas, históricas do saber e da prática de enfermagem”, é possível afirmar que suas teses, desde que foi adotado o referido eixo, têm reiterado a vocação do programa para o desenvolvimento do saber e fazer da enfermagem, com pesquisas desenvolvidas nas diferentes temáticas e correntes de pensamento.

\section{CONSIDERAÇÕES FINAIS}

Se, fazer ciência é buscar a maior aproximação possível da realidade, de forma que as explicações sistemáticas da natureza e da subjetividade da experiência humana sejam compreensíveis aos olhos de todos, pode-se dizer que as teses do Inter, têm contribuído para a construção da ciência que fundamenta a prática da enfermagem e da saúde.

No conjunto, tanto as temáticas como os objetos de estudo e seus resultados, apontam para uma produção com a tendência de persistir e consolidar o movimento de articulação interdisciplinar em sua concepção teórica e metodológica, bem como, de aplicação dos resultados das pesquisas, uma vez que esses, transcendem seu uso apenas na prática da enfermagem. Trazem propostas e apontam diretrizes políticas para o setor saúde e para a formação profissional, assim como, aprofunda a discussão epistemológica do saber da enfermagem.

Apesar de obtermos subsídios importantes e interessantes, por meio da análise dos resumos das teses, para afirmar a importância que essa produção, do ponto de vista teórico, atinge para o meio acadêmico, ainda há o que se pesquisar para comprovar a efetividade dos resultados na prática, em todos as áreas implicadas nas temáticas desenvolvidas, seja na dimensão da organização, da assistência e da educação em enfermagem ${ }^{(11)}$.

Os resultados apresentados nesse estudo expressam a consolidação do espaço da pós-graduação como importante centro de desenvolvimento de pesquisa e pesquisadores, necessitando, no entanto de estudos mais aprofundados sobre o real impacto dos resultados das pesquisas na sociedade e também, do impacto dos pesquisadores formados no perfil da atividade da investigação científica em seus locais de origem. 
(1) Carvalho AC. Associação Brasileira de Enfermagem 1926 - 1976: documentário. Rio de Janeiro: Folha Carioca; 1976.

(2) Nogueira MJ. A pesquisa em enfermagem no Brasil: retrospectiva histórica. Rev Bras Enferm 1982; 16(1):17-26.

(3) Trentini M. A relação entre teoria, pesquisa e prática. Rev Esc Enferm USP 1987; 21(2):135-43.

(4) Coordenação de Aperfeiçoamento de Pessoal de Nível Superior (CAPES). Mestrados e doutorados reconhecidos. Apresenta dados dos programas de pós-graduação por área do conhecimento. [on-line]. Brasília: CAPES; 2004. Disponível em: <http:// www.capes.gov.br/> (21 nov. 2004)

(5) Universidade de São Paulo. Fênis web. Sistema de pós-graduação da USP na internet. [on-line]. Disponível em:http://sistemas1.usp.br:8080/ (18 dez. 2004)

(6) Coordenação de Aperfeiçoamento de Pessoal de Nível Superior (CAPES). Reformulação do sistema de avaliação da pós-graduação: o modelo a ser implantado na avaliação de 1998: documento em discussão. Brasília; 1998.
(7) Oliveira EB, Lisboa MTL. Análise da produção científica da vertente saúde do trabalhador de enfermagem: subjetividade e trabalho. Rev Enferm UERJ 2004; 12(1):24-9.

(8) Bergman R. Conceptualization: knowledge and logie guiding empirical research. Nurs Res 1977; 26(5):924.

(9) Sauer JL. Using a phenomenological research method to study nursing phenomena. In: Leininger MM, editor. Qualitative research methods in nursing. New York: Grune \& Strattton; 1985. p. 93-107.

(10) Angerami ELS. O mister da investigação do enfermeiro. Rev Lat Am Enferm 1993; 1(1):11-22.

(11) Associação Brasileira de Enfermagem (ABEn) Áreas e linhas de pesquisa da enfermagem. [online] Brasília; 2001. Disponível em:http://www. aben nacional.org.br/eee.php (12 ago. 2004)
Programa Interunidades de Doutoramento em Enfermagem: 21 anos construindo ciência 